\title{
Development, Optimization, and Characterization of PEGylated Nanoemulsion of Prostaglandin E1 for Long Circulation
}

\author{
Ying Cheng, ${ }^{1}$ Miao Liu, ${ }^{1}$ Huijing Hu, ${ }^{2}$ Daozhou Liu, ${ }^{1}$ and Siyuan Zhou, ${ }^{1,3}$
}

Received 30 March 2015; accepted 8 July 2015; published online 21 July 2015

\begin{abstract}
Lipo-PGE1 is the most widely used formulation of PGE1 in clinic. However, PGE1 is easier to leak out from lipo-PGE1 and this will lead to the phlebophlogosis when intravenous injection. The stability of lipo-PGE1 in storage and in vivo is also discounted. The aim of this study is to develop a long-circulating prostaglandin E1-loaded nanoemulsion modified with 1,2-distearoyl-sn-glycero-3phosphoethanolamine-N-[methoxy(polyethylene glycol)-2000] (DSPE-PEG) to improve the stability and pharmacokinetics profiles of lipo-PGE1. PEGylated PGE1 nanoemulsion was prepared using a dispersing-homogenized method. The stability of nanoemulsion in 1 month was investigated. Pharmacokinetic studies were employed to evaluate the in vivo profile of the optimized nanoemulsion. The optimized nanoemulsion PGE1-PEG2000(1\%)-NE showed an oil droplet size $<100 \mathrm{~nm}$ with a surface charge of $-14 \mathrm{mV}$. Approximately, $97 \%$ of the PGE1 was encapsulated in the nanoemulsion. The particle size, zeta potential, and drug loading of PGE1-PEG2000(1\%)-NE were stable in 1 month. After PGE1PEG2000(1\%)-NE was intravenously administered to rats, the area under curve (AUC) and half-life of PGE1 were, respectively, 1.47-fold and 5.98-fold higher than those of lipo-PGE1 (commercial formulation). PGE1-PEG2000(1\%)-NE was an ideal formulation for prolonging the elimination time of PGE1. This novel parenteral colloidal delivery system of PGE1 has a promising potential in clinic use.
\end{abstract}

KEY WORDS: LC-MS/MS; nanoemulsion; pharmacokinetic; polyethylene glycol; prostaglandin E1.

\section{INTRODUCTION}

Prostaglandin E1 (PGE1), also known as alprostadil, is an autacoid drug. PGE1 has multiple effects on peripheral vascular system, including increase of peripheral blood flow (1) and viscosity (2), modulation of fibrinolytic system (3), inhibition of platelet aggregation (4), vasodilation, cytoprotection, and angiogenesis. In clinic, PGE1 is used for treatment many diseases such as peripheral arterial occlusive disease (5-8), pulmonary arterial hypertension $(9,10)$, hepatopathy, hypertension, diabetic neuropathy, hypoxia/reperfusion injury (11). In addition, it was found that PGE1 had potential in treatment of mixed arterial and venous ulcers of the lower limbs (12). However, the clinical application of PGE1 is limited due to its low solubility and low bioavailability. PGE1 also has a short elimination half-life because of the first-pass metabolism in the lungs $(13,14)$. Thus, it is difficult for PGE1 to keep a high enough concentration in blood even if it is administered at high doses.

A number of new alternative dosage forms of PGE1, such as lipid microspheres (15), inhaled PLGA particles (16-18) for

\footnotetext{
${ }^{1}$ Department of Pharmaceutics, School of Pharmacy, Fourth Military Medical University, Xi'an, 710032, China.

${ }^{2}$ Xi'an Libang Zhaoxin Biological Technology Co., Ltd., Xi'an, 710061, China.

${ }^{3}$ To whom correspondence should be addressed. (e-mail: zhousy@fmmu.edu.cn)
}

pulmonary arterial hypertension, and nanoparticles (19-22), can prevent PGE1 from inactivation in blood and improve the efficacy of PGE1. Among these new dosage forms, PGE1 lipid microspheres (lipo-PGE1) were established by Mizushima (15). Since it can deliver the encapsulated PGE1 efficiently to disease sites, lipo-PGE1 is used widely in clinic. However, PGE1 is easier to leak out from lipo-PGE1 because of hydrophilic nature of PGE1 (18). This not only leads to the phlebophlogosis when it is intravenously administered but also decreases the stability of lipo-PGE1 in vitro and in vivo.

Nanoemulsion is heterogeneous system composed of oil droplets dispersed in aqueous media and stabilized by using surfactant molecules. Nanoemulsion is suitable to encapsulate and deliver hydrophobic drugs. Besides, nanoemulsion is kinetically stable without any apparent flocculation or coalescence during the long-term storage due to their nanometersized droplets (23-26). The organic solvent is usually used during the preparation of nanoparticle and liposome. Thus, compared with nanoparticle and liposome, the preparation of nanoemulsion is convenient and safe $(27,28)$. At present, the US Food and Drug Administration has approved several nanoemulsion formulation of compound with poor water solubility for clinical use, such as cyclosporin (Neoral®, Gengraf ${ }^{\circledR}$ ) and ritonavir (Norvir $\left.{ }^{\circledR}\right)$.

The factors that limit the systemic exposure of oil-inwater nanoemulsion following their intravenous administration include a rapid clearance from the blood by mononuclear macrophage system (MPS), mainly by Kupffer cells in the liver 
and spleen. Polyethylene glycol (PEG) has been used in many kinds of drug delivery system. PEGylation of nanoemulsion (PEG-NE), which can avoid MPS uptake and prolong the circulation time of the nanoemulsion, is an attractive method to increase systemic exposure of nanoemulsion $(29,30)$.

The goal of this study was to prepare a PEGylated PGE1 nanoemulsion (PGE1-PEG-NE) to improve the pharmacokinetic profile of PGE1 after it was intravenously administered. The PEGylated nanoemulsion, which was composed of glycerol, soybean oil, lecithin, and PGE1, was prepared by a solvent diffusion method in an aqueous system. 1,2-distearoyl-snglycero-3-phosphoethanolamine-N-[methoxy(polyethylene glycol)-2000] (DSPE-PEG) was incorporated into nanoemulsion to obtain PEGylated nanoemulsion. The amount of the DSPEPEG added into the nanoemulsion was optimized according to the size, zeta potential, and drug encapsulation efficiency of the PEGylated nanoemulsion.

Although PGE1 has been already formulated as a commercial injectable emulsion (31), its pharmacokinetic characteristics are generally lacking. It is known that the nanoemulsion system usually affects the pharmacokinetic behavior of the encapsulated drug and consequently changes its therapeutic effect. Thereafter, the pharmacokinetics of the commercial lipid injectable emulsion of PGE1 (lipo-PGE1) and the PEGylated nanoemulsion of PGE1 (PGE1-PEG-NE) were investigated.

\section{EXPERIMENTAL}

\section{Materials}

Prostaglandin (PG) E1 was purchased from Jilin Yinglian Biopharmaceutical Co., Ltd. (Jilin, PR China, Lot No.20120401), internal standard prednisolone was purchased from Sigma. Methanol and acetonitrile were high-performance liquid chromatography (HPLC) grade and obtained from Fisher Scientific (FairLawn, NJ, USA). 1,2-Distearoyl-snglycero-3-phosphoethanolamine-N-[methoxy(polyethylene glycol)-2000] (DSPE-PEG2000) and DSPE-PEG5000 was purchased from Corden Pharma International (Plankstadt, Germany). DSPE-PEG10000 was purchased from Nanocs (New York, USA). Ultrahigh purity water, prepared by using the Milli-Q system, was used throughout the study. All other chemicals were analytical grade and used without further purification. Alprostadil lipid microspheres (lipo-PGE1) (1 mL:5 $\mu \mathrm{g}$ ) were purchased from Xi'an Libang Pharmaceutical Co., Ltd., (Xi'an, PR China).

\section{Preparation and Optimization of the PGE1 PEGylated Nanoemulsion}

The oil and the aqueous phases were first separately prepared. The oil phase, consisting of soybean oil and egg lecithin, was heated at $70^{\circ} \mathrm{C}$ under stirring to ensure lecithin was completely dissolved. The obtained oil phase was cooled down to the room temperature $\left(25^{\circ} \mathrm{C}\right)$. Then, PGE1 and different types and amounts of the DSPE-PEG (as shown in Table I) were added and dissolved in the oil phase. The oil phase was stored at $25^{\circ} \mathrm{C}$. The aqueous phase was prepared by dissolving sodium oleate in water. To adjust isotonicity, glycerol $(2.5 \%, w / w)$ was added to the aqueous phase. The phases were combined by adding the oil phase to the aqueous phase
Table I. The Type and Amount of the DSPE-PEG Added in the Different Formulation

\begin{tabular}{lll}
\hline Formulation number & Type of DSPE-PEG & $\begin{array}{l}\text { Amount of } \\
\text { DSPE-PEG }\end{array}$ \\
\hline PGE1-PEG2000(0.5\%)-NE & DSPE-PEG2000 & $0.5 \%$ \\
PGE1-PEG2000(1\%)-NE & DSPE-PEG2000 & $1 \%$ \\
PGE1-PEG2000(2\%)-NE & DSPE-PEG2000 & $2 \%$ \\
PGE1-PEG5000(0.5\%)-NE & DSPE-PEG5000 & $0.5 \%$ \\
PGE1-PEG5000(1\%)-NE & DSPE-PEG5000 & $1 \%$ \\
PGE1-PEG10000(0.5\%)-NE & DSPE-PEG10000 & $0.5 \%$ \\
\hline
\end{tabular}

$D S P E-P E G$ 1,2-distearoyl-sn-glycero-3-phosphoethanolamine-N[methoxy(polyethylene glycol)-2000]

and further pre-homogenized with a high-shear dispersing emulsifier (FLUKO FA25, FLUKO company, Germany) at $10,000 \mathrm{rpm}$ for $5 \mathrm{~min}$. The obtained coarse emulsion was subsequently homogenized with a high-pressure homogenizer (PandaPlus1000; Niro Soavi Company, Italy) at 800 bar. The high-pressure homogenization process was repeated 6 cycles discontinuously.

\section{Characterization of PGE1-PEG-NE and Lipo-PGE1}

The zeta potential, average particle size, and size distribution of lipo-PGE1 and PGE1 PEGylated nanoemulsion (PGE1-PEG-NE) with different formulation were determined by dynamic light scattering using a Beckman Coulter particle analyzer (Fullerton, California, USA). Samples were diluted with ultrahigh purity water before analysis.

\section{Encapsulation Efficiency Evaluation}

The free PGE1 and PGE1 encapsulated in PGE1-PEG$\mathrm{NE}$ and the lipo-PGE1 were separated by ultra-filtration. Briefly, the sample $(1 \mathrm{~mL})$ was placed in the Centrisart filter (molecular weight cutoff $5000 \mathrm{kDa}$, Sartorius, AG, Germany) and centrifuged at $8000 \times g$ for $10 \mathrm{~min}$. The nanoemulsion with encapsulated PGE1 remained in the outer chamber, and aqueous phase containing free PGE1 was moved into the sample recovery chamber. The amount of the PGE1 in both phases was determined by using liquid chromatography-mass/mass (LC-MS/MS) method as described below, and encapsulation efficiency was calculated.

\section{Stability of PGE1-PEG-NE}

To evaluate the physical stability of lipo-PGE1 and PGE1-PEG-NE with different formulations, they were stored at room temperature for 1 month. The average particle size, size distribution, surface charge, and encapsulation efficiency of lipo-PGE1 and PGE1-PEG-NE with different formulations were determined by using the previously described method.

\section{In Vivo Pharmacokinetic Behavior}

All animal procedures were approved by the Animal Ethics Committee of the Fourth Military Medical University. 
Adult male Sprague-Dawley rats $(200 \pm 20 \mathrm{~g})$ were obtained from the Experimental Animal Center of Fourth Military Medical University, Xi'an, Shaanxi. The rats had free access to food and water. The animal room was maintained on a 12-h light/dark cycle, with a temperature range between 20 and $22^{\circ} \mathrm{C}$ and $50 \%$ relative humidity.

The optimized PGE1-PEG-NE was freshly prepared and administered to rats via the tail vein at the dose of $200 \mu \mathrm{g}$ PGE1/kg $(n=6)$. The lipo-PGE1 was also administered to rats at the same route and dose. Blood was collected at pre-dose and 1, 2, 4, 6, 10, 15, 20,30, 60 min after the administration of nanoemulsion. The plasma was obtained by centrifugation of blood at $500 \times g\left(4^{\circ} \mathrm{C}, 10 \mathrm{~min}\right)$. The concentration of PGE1 in plasma was detected by LC-MS/MS.

\section{Sample Preparation and LC-MS/MS Method}

PGE1 and IS (prednisolone) in plasma were extracted using a liquid-liquid extraction method. Briefly, plasma samples $(100 \mu \mathrm{L})$, IS (prednisolone, $10 \mu \mathrm{L}, 100 \mathrm{ng} / \mathrm{mL}$ ), and diethyl ether $(2 \mathrm{~mL})$ were added into a clear tube. The mixture was then vortexed for $3 \mathrm{~min}$ and centrifuged $(1000 \times g$ for $10 \mathrm{~min})$. The organic layer was carefully transferred to a fresh tube (about $1.5 \mathrm{~mL}$ ) and was evaporated to dryness by using Termovap sample concentrator $\left(35^{\circ} \mathrm{C}, 7 \mathrm{~min}\right)$. The residue in the tube was dissolved in methanol $(100 \mu \mathrm{L})$ for LC-MS/MS analysis.

A Quattro Premier liquid chromatography-mass/mass (LC-MS/MS) system (Waters Corp., Milford, MA) operating under MassLynx 4.1 software was used. An XTerra C18 analytical column $(150 \times 2.1 \mathrm{~mm}, 5 \mathrm{~mm}$, Waters Corp. $)$ was used. The mobile phase consisted of acetonitrile- $0.1 \%$ formic acid in water $(80: 20, v / v)$ at a flow rate of $0.2 \mathrm{~mL} / \mathrm{min}$. An electrospray ionization-mass spectrometry (ESI) source was used and was operated in negative ion mode in selected multiple reaction monitoring (MRM) mode. The source temperature was $110^{\circ} \mathrm{C}$, and the desolvation temperature was $300^{\circ} \mathrm{C}$. Nitrogen was used as the desolvation and cone gas with a flow rate of 650 and $50 \mathrm{~L} / \mathrm{h}$, respectively. Argon was used as the collision gas at a rate of $0.18 \mathrm{~mL} / \mathrm{min}$. The autosampler was maintained at $25^{\circ} \mathrm{C}$ and the injection volume was $10 \mu \mathrm{L}$. Ionic reaction was $352.9 \rightarrow 317.0(\mathrm{~m} / z)$ for PGE1 and $359.0 \rightarrow 329.0$ $(\mathrm{m} / \mathrm{z})$ for IS. Collision energy was -30 and $-25 \mathrm{eV}$ for PGE1 and IS, respectively.

\section{Data Analysis}

The pharmacokinetic parameters were calculated by using non-compartment model by the pharmacokinetic program Drug and Statistics, version 3.0 (Mathematical Pharmacology Professional Committee of China, Shanghai, China). The highest observed plasma concentration and its corresponding sampling time were defined as Cmax and Tmax, respectively. The area under the curve from time zero to infinity $\left(\mathrm{AUC}_{0-\infty}\right)$ and to the last detectable time point $\left(\mathrm{AUC}_{0-T}\right)$ was calculated by the linear trapezoidal method. The terminal half-life $\left(t_{1 / 2}\right)$ was estimated by a linear regression analysis of the three or four data points of the terminal linear segment of the log plasma concentration versus time curve. Plasma clearance (CL) and volume of distribution values were calculated by standard methods.

\section{Statistical Analysis}

Data were reported as mean \pm standard deviation of means (SD). A minimum of three animals per group was used. The significances of differences were determined using Student's $t$ test two-tailed for each paired experiment. $p<0.05$ was considered statistically significant in all cases.

\section{RESULTS}

\section{Validation of Analytical Method}

The typical chromatograms to detect PGE1 in rat plasma are shown in Fig. 1. A standard curve was constructed by plotting the ratio of peak areas of PGE1 and IS versus PGE1 concentration. The correlation coefficient of PGE1 was found to be 0.9947 for plasma samples. The mean \pm standard deviations ( $n=3$ replicates) of PGE1 slope and intercept of the regression curve were 5.515 and 4.730 for plasma. Calibration curve was linear over the concentration range of $1-500 \mathrm{ng} / \mathrm{mL}$. The limit of detection (LOD) $(\mathrm{S} / \mathrm{N}>4)$ and limit of quantitation (LOQ) $(\mathrm{S} / \mathrm{N}>10)$ were 0.3 and $1 \mathrm{ng} / \mathrm{mL}$, respectively. The calibration standards for intraday and interday accuracy and precision are shown in Table II. The intraday and interday precisions ranged from 5.6 to $7.0 \%$ and from 3.9 to $6.5 \%$, respectively. The above data implied that the analytical method was highly sensitive and specific and was suitable to the pharmacokinetics study of PGE1 in rats.

\section{Preparation and Characterization of Nanoemulsions}

The nanoemulsions were prepared by using different molecular weights of DSPE-PEG as well as different concentrations of DSPE-PEG. The encapsulation efficiency, zeta potential, average particle size, and size distribution of different formulations and lipo-PGE1 are shown in Table III. The results indicated when content of PEG-DSPE2000 increased from 0.5 to $1 \%$, average particle size of PGE1-PEG2000-NE became smaller with the increase of content of PEGDSPE2000, and the zeta potential of PGE1 nanoemulsion decreased significantly with the increase of content of PEGDSPE2000. When the content of PEG-DSPE2000 was beyond $2 \%$, average particle size and zeta potential of PGE1 nanoemulsion increased obviously. The average particle size of PGE1 nanoemulsion increased significantly with the increase of molecular weight of PEG in DSPE-PEG. The zeta potential of PGE1 nanoemulsion decreased significantly with the increase of molecular weight of PEG in DSPE-PEG. There was no significant effect of type and amount of the DSPE-PEG on the encapsulation efficiency.

\section{Stability Studies}

The physical stability of lipo-PGE1 and PGE1-PEG-NE with different formulations in 1 month of storage at $25^{\circ} \mathrm{C}$ was investigated. The results are shown in Table III. The encapsulation efficiency, droplet size, and surface charge are considered to be the most representative parameters in the control of nanoemulsion stability. The results showed there was no significant change $(p<0.05)$ in encapsulation efficiency, average particle size, and surface charge after PGE1- 


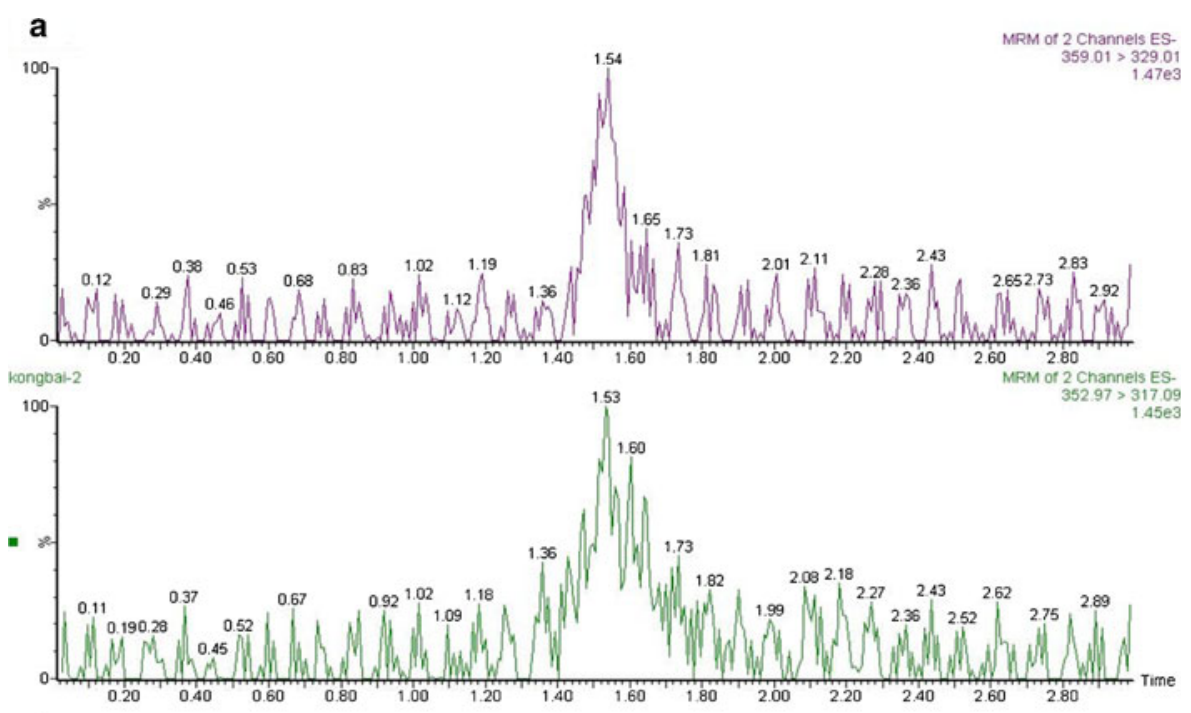

$$
\text { b }
$$
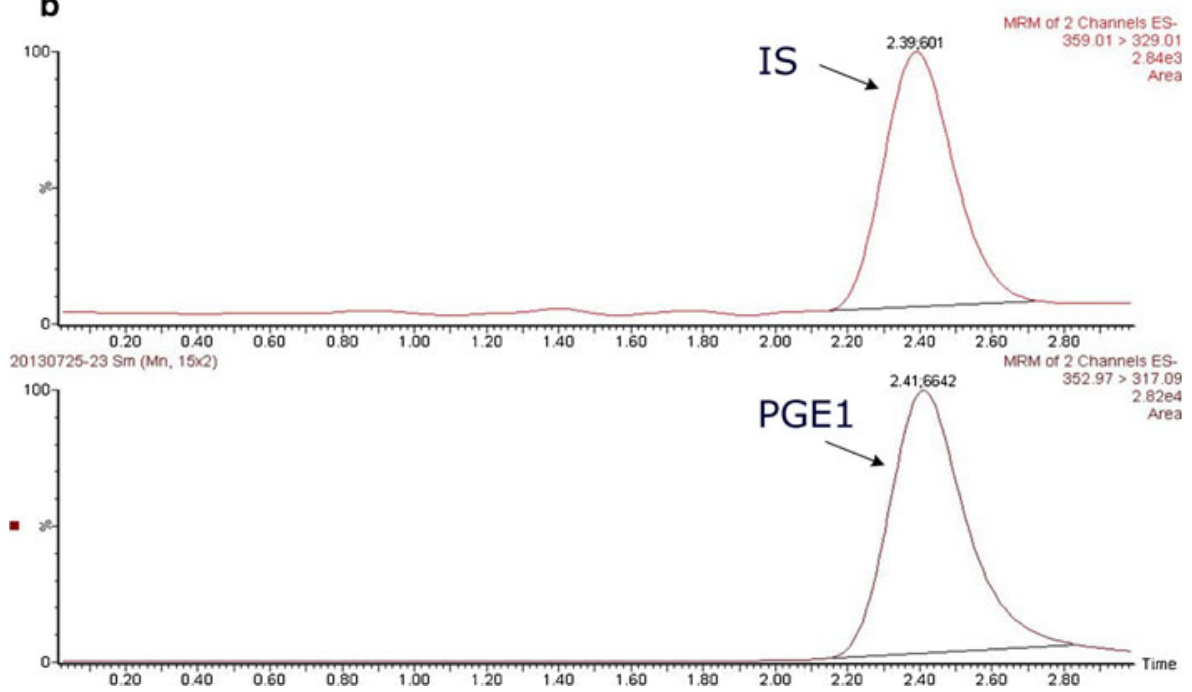

Fig. 1. Typical chromatograms of drug-free rat plasma (a) and spiked rat plasma containing $100 \mathrm{ng} /$ $\mathrm{mL}$ of PGE1 and $100 \mathrm{ng} / \mathrm{mL}$ of IS (b)

PEG2000(1\%)-NE, and lipo-PGE1 was stored for 1 month as compared with initial samples at 0 day. This implied that PGE1-PEG2000(1\%)-NE was stable in 1 month. Although the average particle size and surface charge of PGE1PEG2000(0.5\%)-NE, PGE1-PEG2000(2\%)-NE, PGE1PEG5000(0.5\%)-NE, PGE1-PEG5000(1\%)-NE, and PGE1-

Table II. Intra- and Interday Precision and Accuracy of Analytical Method to Detect PGE1 in Rat Plasma

\begin{tabular}{|c|c|c|c|c|c|}
\hline & & $\begin{array}{l}\text { Nominal concentration } \\
\quad(\mathrm{ng} / \mathrm{mL})\end{array}$ & Measured $($ mean $\pm \mathrm{SD})$ & Precision RSD (\%) & Accuracy (\%) \\
\hline \multirow[t]{9}{*}{ Intraday } & \multirow[t]{3}{*}{ Day 1} & 2 & $1.954+0.124$ & 6.34 & 95.3 \\
\hline & & 50 & $49.06 \pm 3.22$ & 6.49 & 97.1 \\
\hline & & 400 & $399.0 \pm 21.7$ & 4.66 & 103.5 \\
\hline & \multirow[t]{3}{*}{ Day 2} & 2 & $1.922 \pm 0.129$ & 6.74 & 96.1 \\
\hline & & 50 & $48.93 \pm 1.95$ & 3.88 & 97.9 \\
\hline & & 400 & $415.0 \pm 22.3$ & 5.35 & 103.9 \\
\hline & \multirow[t]{3}{*}{ Day 3} & 2 & $1.932 \pm 0.123$ & 6.37 & 96.6 \\
\hline & & 50 & $51.79 \pm 2.58$ & 4.99 & 103.5 \\
\hline & & 400 & $387.4 \pm 17.5$ & 4.59 & 96.8 \\
\hline \multirow[t]{3}{*}{ Interday } & & 2 & $1.921 \pm 0.108$ & 5.64 & 96.0 \\
\hline & & 50 & $49.76 \pm 3.46$ & 6.95 & 99.5 \\
\hline & & 400 & $405.7 \pm 23.4$ & 5.76 & 101.4 \\
\hline
\end{tabular}


Table III. Physicochemical Characteristics of Lipo-PGE1 and PGE1-PEG-NE

\begin{tabular}{|c|c|c|c|c|c|}
\hline Formulation & Time (day) & Size $(\mathrm{nm})$ & PDI & Zeta potential $(\mathrm{mV})$ & $\mathrm{EE}(\%)$ \\
\hline \multirow[t]{2}{*}{ Lipo-PGE1 } & 0 & $172 \pm 5$ & $0.135 \pm 0.014$ & $-26.0 \pm 1.3$ & $98.3 \pm 0.7$ \\
\hline & 30 & $178 \pm 6$ & $0.141 \pm 0.012$ & $-27.3 \pm 1.5$ & $97.2 \pm 0.7$ \\
\hline \multirow[t]{2}{*}{ PGE1-PEG2000(0.5\%)-NE } & 0 & $99 \pm 6$ & $0.161 \pm 0.007$ & $-20.1 \pm 3.0$ & $97.9 \pm 0.6$ \\
\hline & 30 & $102 \pm 7$ & $0.158 \pm 0.010$ & $-21.5 \pm 2.9$ & $95.2 \pm 0.5^{* *}$ \\
\hline \multirow[t]{2}{*}{ PGE1-PEG2000(1\%)-NE } & 0 & $83 \pm 4$ & $0.155 \pm 0.012$ & $-13.7 \pm 1.6$ & $97.9 \pm 0.2$ \\
\hline & 30 & $86 \pm 5$ & $0.159 \pm 0.011$ & $-14.5 \pm 1.8$ & $97.5 \pm 0.5$ \\
\hline \multirow[t]{2}{*}{ PGE1-PEG2000(2\%)-NE } & 0 & $115 \pm 4$ & $0.180 \pm 0.010$ & $-17.9 \pm 1.8$ & $97.5 \pm 0.3$ \\
\hline & 30 & $120 \pm 6$ & $0.184 \pm 0.009$ & $-18.4 \pm 2.1$ & $95.7 \pm 0.3^{*}$ \\
\hline \multirow[t]{2}{*}{ PGE1-PEG5000(0.5\%)-NE } & 0 & $143 \pm 6$ & $0.177 \pm 0.015$ & $-15.8 \pm 1.5$ & $98.2 \pm 0.5$ \\
\hline & 30 & $147 \pm 7$ & $0.179 \pm 0.014$ & $-14.7 \pm 2.4$ & $96.8 \pm 0.5^{*}$ \\
\hline \multirow[t]{2}{*}{ PGE1-PEG5000(1\%)-NE } & 0 & $166 \pm 5$ & $0.180 \pm 0.015$ & $-10.5 \pm 1.4$ & $98.0 \pm 0.2$ \\
\hline & 30 & $171 \pm 7$ & $0.182 \pm 0.016$ & $-9.6 \pm 1.8$ & $96.1 \pm 0.5^{* *}$ \\
\hline \multirow[t]{2}{*}{ PGE1-PEG10000(0.5\%)-NE } & 0 & $201 \pm 9$ & $0.174 \pm 0.016$ & $-7.8 \pm 0.7$ & $97.7 \pm 0.6$ \\
\hline & 30 & $210 \pm 11$ & $0.172 \pm 0.013$ & $-7.0 \pm 0.7$ & $95.6 \pm 0.8^{*}$ \\
\hline
\end{tabular}

Data are mean \pm SD $(n=3)$

$P D I$ polydispersity index, $E E$ encapsulation efficiency

$* p<0.05 ; * * p<0.01 v s$ the same formulation at 0 day

PEG10000(1\%)-NE did not change significantly after they were stored for 1 month as compared with initial samples at 0 day, their encapsulation efficiency decreased obviously after they were stored for 1 month as compared with initial samples at 0 day. According to the average particle size, zeta potential, and stability, PGE1-PEG2000(1\%)-NE was selected for further study. The typical TEM image of PGE1-PEG2000(1\%)$\mathrm{NE}$ and its size distribution measured by DLS at room temperature are shown in Fig. 2.

\section{In Vivo Pharmacokinetic Behavior}

Figure 3 shows the plasma concentration versus time profiles of PGE1 after PGE1-PEG2000(1\%)-NE and lipoPGE1 were intravenously administered. The pharmacokinetic parameters are shown in Table IV. The plasma concentration and AUC of PGE1-PEG2000(1\%)-NE were significantly higher as compared with the same dose of lipo-PGE1. The Cmax of PGE1-PEG2000(1\%)-NE was higher than that of lipo-PGE1. Additionally, compared with lipo-PGE1, the elimination half-life of PGE1-PEG2000(1\%)-NE was significantly longer. After PGE1-PEG2000(1\%)-NE was intravenously administered, the total plasma PGE1 clearance was $0.1660 \mathrm{~L} / \mathrm{min} / \mathrm{kg}$. The total plasma PGE1 clearance was $0.2417 \mathrm{~L} / \mathrm{min} / \mathrm{kg}$ after the same dose of lipo-PGE1 was intravenously administered. Compared with lipo-PGE1, the apparent volume of distribution of PGE1 was significantly increased after PGE1-PEG2000(1\%)-NE was intravenously administered. Compared with the lipo-PGE1, long distribution half-life was observed after the same dose of PGE1-PEG2000(1\%)-NE was intravenously administered.

\section{DISCUSSION}

In recent years, nanoemulsions have caused much attention as a feasible carrier for the delivery of hydrophobic drugs. Their high solubilization capacity, ease of production, and long-term stability make nanoemulsions as promising drug delivery systems (32). The droplet size of nanoemulsions is an important factor because it influences drug-release

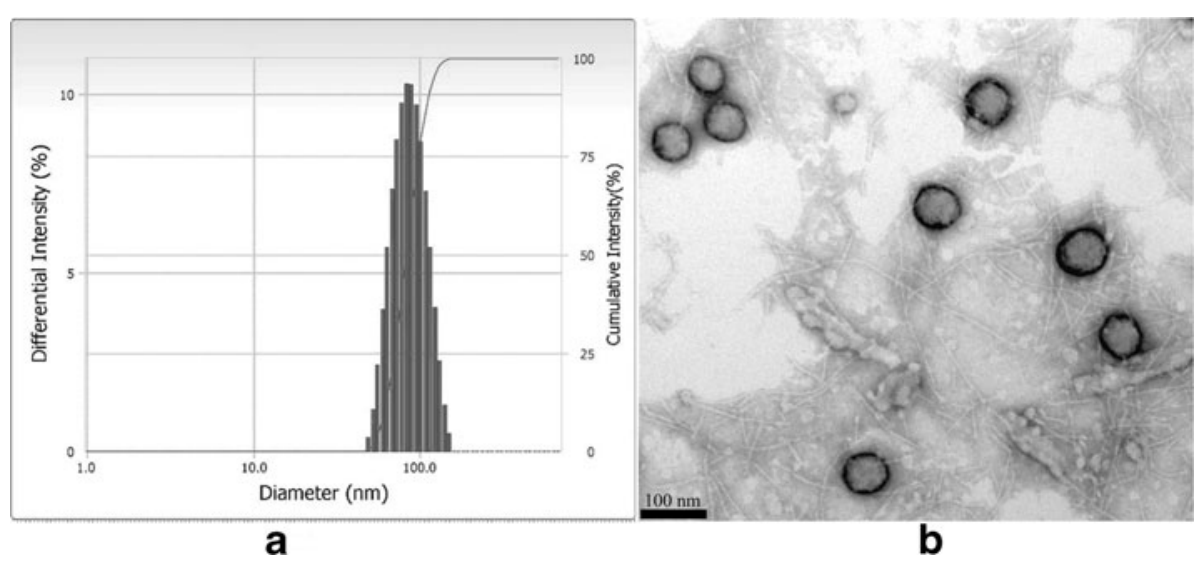

Fig. 2. Typical size distribution measured by DLS (a) and TEM (b) image of PGE1-PEG2000(1\%)-NE at room temperature. Abbreviations: TEM transmission electron microscopy, DLS dynamic light scattering 


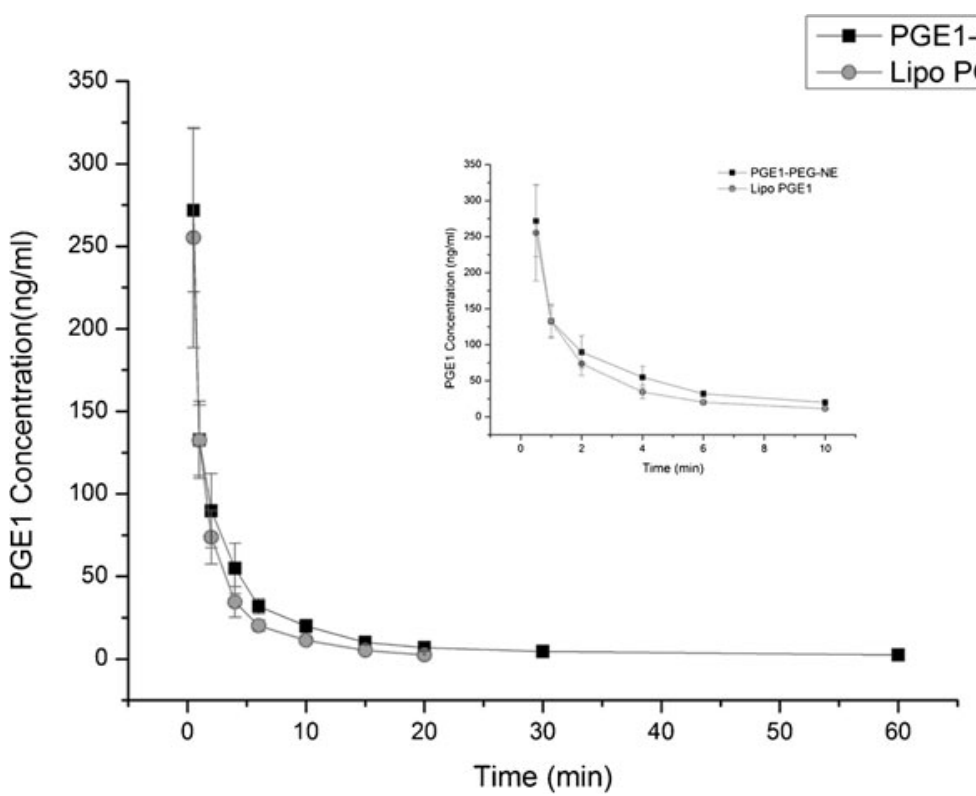

Fig. 3. Pharmacokinetics profile of PGE1 after $200 \mu \mathrm{g} / \mathrm{kg}$ formulation was intravenously administered to rats $(N=6$, mean $\pm \mathrm{SD})$. The inset plasma $v s$ time graph represents the pharmacokinetics characteristics of PGE1 in 10 min after formulation was intravenously administered to rats

behavior and stability (33). For a nanoparticle to exhibit prolonged circulation and enhanced permeability and retention (EPR) effect, the smallest average particle size is $5.5 \mathrm{~nm}$, the renal filtration cutoff size (34). Particles that size are smaller than $50 \mathrm{~nm}$ will interact with hepatocytes. Large particles $(>1 \mu \mathrm{m})$ may cause emboli (the diameter of the smallest blood capillaries is $4 \mu \mathrm{m}$ ) and can be taken up by mononuclear phagocytic system (MPS) (35). Fang et al. (36) reported that the protein adsorption on the 80 -nm particles $(6 \%)$ was lower than that on larger sizes (171 and $243 \mathrm{~nm}, 23$ and $34 \%$, respectively) because smaller particles exhibit a higher surface density of PEG. The net charge on a surface of a particle is an

Table IV. Pharmacokinetic Parameters of PGE1-PEG2000(1\%)-NE and Lipo-PGE1 after They Were Intravenously Administered to Rats

\begin{tabular}{|c|c|c|}
\hline Parameters & PGE1-PEG2000(1\%)-NE & Lipo-PGE1 \\
\hline$t_{1 / 2 \alpha}(\min )$ & $2.021 \pm 0.508 * *$ & $0.696 \pm 0.365$ \\
\hline$t_{1 / 2 \beta}(\min )$ & $30.03 \pm 15.07 * *$ & $5.1 \pm 1.7$ \\
\hline $\mathrm{V}(\mathrm{L} / \mathrm{kg})$ & $1.050 \pm 0.162 *$ & $0.5809 \pm 0.217$ \\
\hline CL (L/min/kg) & $0.1660 \pm 0.0284 *$ & $0.2417 \pm 0.0439$ \\
\hline $\operatorname{AUC}_{(0-\mathrm{t})}(\mathrm{ug} / \mathrm{L} \times \min )$ & $1012 \pm 140 * *$ & $685.4 \pm 116.5$ \\
\hline $\operatorname{AUC}_{(0-\infty)}(\mathrm{ug} / \mathrm{L} \times \min )$ & $1234 \pm 205^{*}$ & $849.7 \pm 149.2$ \\
\hline$K_{10}(1 / \mathrm{min})$ & $0.159 \pm 0.020 * *$ & $0.459 \pm 0.148$ \\
\hline $\operatorname{MRT}_{(0-\mathrm{t})}(\min )$ & $9.367 \pm 1.783 * *$ & $3.254 \pm 0.339$ \\
\hline $\operatorname{MRT}_{(0-\infty)}(\min )$ & $13.08 \pm 5.59 * *$ & $3.777 \pm 0.748$ \\
\hline $\mathrm{Fr}$ & $147.7 \%$ & \\
\hline
\end{tabular}

$n=6$, data are mean \pm SD

Abbreviations: $S D$ standard deviation, $A U C$ area under curve, $t 1 / 2$ half-life time, $M R T$ mean residence time, $V$ apparent volume of distribution, $\mathrm{Fr}$ relative bioavailability, $D$ dose, $t_{1 / 2 \alpha}$ rapid elimination half-life, $t_{1 / 2 \beta}$ terminal elimination half-life, $C L$ drug clearance, $k_{10}$ rapid elimination rate constant

$* p<0.05 ; * * p<0.01$ vs lipo-PGE1 influential physical factor impacting PK and PD. Generally speaking, negatively charged particles $(\xi \leq 10 \mathrm{mV})$ exhibit strong MPS uptake, and positive particles $(\xi>10 \mathrm{mV})$ will induce serum protein aggregation. Neutral nanoparticles (within $\pm 10 \mathrm{mV}$ ) exhibit the least MPS interaction and the longest circulation (37). Thus, based on stability, zeta potential, and average particle size, the PGE1-PEG2000(1\%)-NE is the most suitable nanoemulsion for the pharmacokinetic study. As the index of size distribution, the PDI represents the similarity between particles. The more closer to zero the PDI value is, the more homogeneous the droplets are (38). A large PDI value indicates that the particles have a broad size distribution and are substantially different in size. The ununiform particle size can cause pharmacokinetic parameters to be irregular and can affect the therapeutic efficiency of a drug formulation (39). PDI value of PGE1-PEG2000(1\%)-NE was below 0.2 , this indicated that the particle size distribution of PGE1-PEG2000(1\%)-NE was homogeneous. This was due to the nanoemulsion ingredients and preparation method that had a process of high-pressure homogenization.

Several polymers, such as polysaccharides, have been used to coat nanoparticles to efficiently increase circulation time (40). Among the various polymers, polyethylene glycol (PEG) is the most widely used strategy to create a steric barrier on the surface of nanoparticles to block the absorption of blood protein $(41,42)$. Poly(ethylene glycol) (PEG) was first introduced in the early 1990s to modify the surface of liposomes to improve pharmacokinetics (PK) after liposomes were intravenously (i.v.) administered (43). Binding of plasma proteins is the primary mechanism for the MPS to recognize the nanoparticles in blood circulation, causing a major loss of the injected dose (ID) $(>50 \%)$ within a few hours after i.v. injection (44). PEGylated nanoparticles are often referred as "stealth" nanoparticles because they can escape the surveillance of MPS better than the control nanoparticles. PEGylation is also a key factor, influences 
physicochemical properties of nanoparticles, and consequently affects the in vivo cycle time of the nanoparticle (45). In the molecule of PEG-DSPE, the hydrophilic part is larger as in comparison with its double hydrophobic fatty acid tail. There is mushroom-like and brush-like configuration of PEG when PEG is incorporated in the surface of the nanoemulsion or nanoparticles (45). When the density was low and the chain length was short, the PEG is mushroom-like, and the curvature of the membrane increased, which resulted in the decrease of the PGE1-PEG2000(1\%)-NE diameter. When the PEG density increased and there was no more room for the curvature of the membrane, the PEG was brush-like configuration, which led to the increase of diameter. The molecular weight of PEG significantly affected the size of PGE1-PEG-NE. The size of PEGDSPE5000 or PEG-DSPE10000 modified nanoemulsion was bigger than PEG-DSPE2000 modified nanoemulsion. This implied that with the increase of molecular weight of PEG, the size of PGE1-PEG-NE became bigger.

The quantification of PGE1 has been achieved by GCMS/MS (46,47), HPLC (48), HPLC-MS/MS (49), and UPLCMS/MS (50), among these methods, the UPLC-MS/MS, which is the most sensitive and high throughputs, is suitable to be applied to pharmacokinetic studies. However, the instrument is so expensive that the method is not suitable for every lab. We developed a LC-MS/MS assay for the measurement of the PGE1 amount encapsulated in the nanoemulsions and PGE1 concentrations in rat plasma. The method had been proved to be fast, accurate, and reliable for its applications. In this method, we used the short chromatography column because the signal of the PGE1 was more stable and sensitive by using short column. Although the retention time of PGE1 and IS was near similar, there was no disturbance on the detection of the PGE1 and IS due to the high selectivity of the MRM scan mode. Different extraction methods including protein precipitation (methanol, ethanol, and acetonitrile) and liquid-liquid extraction using ethyl acetate and diethyl ether were tested in our experiment. An optimized diethyl ether-based liquid-liquid extraction method was selected due to its high extraction recoveries and was less time consuming. So, the extraction solvent was diethyl ether. To increase the extraction recovery, the formic acid was added to the plasma. The method we developed is the first LC-MS/MS method used for determining the PGE1 concentration in rat plasma.

A comparative pharmacokinetic study between PGE1PEG2000(1\%)-NE (200 $\mu \mathrm{g} / \mathrm{kg})$ and lipo-PGE1 $(200 \mu \mathrm{g} / \mathrm{kg})$ was performed by determining the concentration of PGE1 in rat plasma for $60 \mathrm{~min}$ after an intravenous administration. The area of the concentration-time curve is currently considered to be the standard method to assess the concentration in blood. In pharmacokinetic studies, the area under the concentrationtime curve is showed to reflect the circulating situation. The AUC of the PGE1 after administration of PGE1PEG2000(1\%)-NE was about 1.47-fold higher than that of lipo-PGE1. PGE1 was detectable in rat plasma at $60 \mathrm{~min}$ after PGE1-PEG2000(1\%)-NE was administered. In contrast, PGE1 was not detectable in rat plasma at $20 \mathrm{~min}$ after lipoPGE1 was administered. The pharmacokinetic parameters suggested that the metabolism of PGE1 in rats was significantly delayed after it was encapsulated into PEGylated nanoemulsion. PGE1-PEG2000(1\%)-NE showed a significant sustained release characteristic. Because of the high AUC of PGE1-PEG2000(1\%)-NE, the dose of the PGE1 could be reduced. This could significantly reduce drug toxicity and increase the therapeutic effect. The PEG was an important factor that affected the pharmacokinetic profile of the PGE1 nanoemulsion formulation. So, the new PGE1-PEG-NE was much more suitable for encapsulating PGE1 than other nanocarriers. PEG chains supply "invisibility" to the drug carrier. This leads to a reduced recognition of the MPS and an extended time of circulation.

In the past several years, some PGE1-loaded delivery systems had been developed as novel intravenous formulations. Tsutomu Ishihara et al. (19) developed nanoparticles that efficiently incorporated PGE1 by blending PLA homopolymers and PEG-PLA block copolymers in the presence of iron. This formulation prevented the inactivation of PGE1, exhibited a long-term therapeutic effect due to slow release along with degradation of the polymers, and controlled the biodistribution to target sites. Yu Gao et al. (22) prepared a lipid nanoparticles loading PGE1 by highpressure homogenization. PLNs exhibited a sustained release with low burst drug release. Lipid nanoparticles could effectively protect PGE1 from degradation and release PLNs sustainedly, which resulted in the improved antiinflammatory effects and reduce the side effect. Miho Takeda et al. (21) prepared PGE1 2-(phosphonooxy)ethyl ester sodium salt (C2), which showed the most efficient hydrolysis to yield PGE1 in human serum. An in vitro platelet aggregation assay showed that C2 inhibited aggregation only after preincubation in serum, suggesting that $\mathrm{C} 2$ is a prodrug of PGE1. C2 released sustainedly from the nanoparticles by using high molecular weight PLA, which is useful in clinic. Mitsuko Takenaga et al. (20) focused on the effect of Nano PGE1on SCI-induced motor dysfunction in a rat model and examined its distribution and the mechanism. The results suggested that nano PGE1 significantly improved hind limb motor dysfunction induced by SCI in rats. The steady level of PGE1 released from nanoparticles, which was accumulated in the injured site by EPR effect, would prevent cell death, induce angiogenesis, and improve blood flow to survive the remaining cells and recruit the function. These nanoparticles all showed good sustained-release profile; however, the preparation process is more complex and the polymer used in the nanoparticle may not be well-tolerated in humans. The PGE1PEG2000(1\%)-NE was prepared by using soybean oil, which have been clinically available for several decades and are currently used in many parenteral formulations. Meanwhile, lecithin (E80) and mPEG-DSPE were welltolerated in humans. Compared with other drug delivery system, PGE1-PEG2000(1\%)-NE had the advantages such as easy production and no organic solvent was used in the preparation.

Finally, PGE1-PEG2000(1\%)-NE could be successfully prepared on a large-scale (20-fold scale), sterilized by high temperature using autoclave sterilizer. During storage of PGE1-PEG2000(1\%)-NE for 1 month at $25^{\circ} \mathrm{C}$, no chemical changes of PGE1 or leaking (burst release) from nanoemulsion were observed. Concerning safety for clinical use, the additives in nanoemulsion seem to be acceptable. 


\section{CONCLUSION}

LC-MS/MS-based analytical method was specific, sensitive, and accurate. PGE1-PEG2000(1\%)-NE showed small PDI, high entrapment efficiency, stability, as well as a significant sustained release characteristic in vivo. Thus, as an alternative parenteral colloidal delivery system, PGE1-PEG2000(1\%)-NE had a promising potential in clinic application.

Conflict of interest The authors declare that they have no competing interests.

\section{REFERENCES}

1. Makino H, Aoki M, Hashiya N, Yamasaki K, Hiraoka K, Shimizu $\mathrm{H}$, et al. Increase in peripheral blood flow by intravenous administration of prostaglandin E1 in patients with peripheral arterial disease, accompanied by up-regulation of hepatocyte growth factor. Hypertens Res. 2004:27(2):85-91.

2. Acciavatti A, Laghi Pasini F, Capecchi PL, Messa GL, Lazzerini PE, De Giorgi L, et al. Effects of alprostadil on blood rheology and nucleoside metabolism in patients affected with lower limb chronic ischaemia. Clin Hemorheol Microcirc. 2001;24(1):49-57.

3. Bandinelli F, Bartoli F, Perfetto E, Del Rosso A, Moggi-Pignone $\mathrm{A}$, Guiducci S, et al. The fibrinolytic system components are increased in systemic sclerosis and modulated by alprostadil (alpha1 ciclodestryn). Clin Exp Rheumatol. 2005;23(5):671-7.

4. Marquis NR, Vigdahl RL, Tavormina PA. Platelet aggregation. I. Regulation by cyclic AMP and prostaglandin E1. Biochem Biophys Res Commun. 1969;36(6):965-72.

5. Hirai M, Nakayama R. Haemodynamic effects of intra-arterial and intravenous administration of prostaglandin E1 in patients with peripheral arterial disease. Br J Surg. 1986;73(1):20-3.

6. Sinzinger H, Virgolini I, O'Grady J. Clinical trials of PGE1, PGI2 and mimetics in patients with peripheral vascular disease. Prog Clin Biol Res. 1989;301:85-96.

7. Thum J, Caspary L, Creutzig A, Alexander K. Intra-arterial and intravenous administration of prostaglandin E1 cause different changes to skin microcirculation in patients with peripheral arterial occlusive disease. Vasa. 1998;27(2):100-5.

8. Weiss T, Eckstein H, Weiss C, Diehm C. Neutrophil function in peripheral arterial occlusive disease: the effects of prostaglandin E1. Vasc Med. 1998;3(3):171-5.

9. Kunimoto F, Arai K, Isa Y, Koyano T, Kadoi Y, Saito S, et al. A comparative study of the vasodilator effects of prostaglandin E1 in patients with pulmonary hypertension after mitral valve replacement and with adult respiratory distress syndrome. Anesth Analg. 1997;85(3):507-13.

10. Shen J, He B, Wang B. Effects of lipo-prostaglandin E1 on pulmonary hemodynamics and clinical outcomes in patients with pulmonary arterial hypertension. Chest. 2005;128(2):714-9. doi:10.1378/chest.128.2.714.

11. Makita S, Nakamura M, Ohhira A, Itoh S, Hiramori K. Effects of prostaglandin E1 infusion on limb hemodynamics and vasodilatory response in patients with arteriosclerosis obliterans. Cardiovasc Drugs Ther. 1997;11(3):441-8.

12. De Caridi G, Massara M, Stilo F, Spinelli F, Grande R, Butrico L, et al. Effectiveness of prostaglandin E1 in patients with mixed arterial and venous ulcers of the lower limbs. Int Wound J. 2014. doi:10.1111/iwj.12334.

13. Cawello W, Schweer H, Dietrich B, Seyberth HW, Albrecht D, Fox A, et al. Pharmacokinetics of prostaglandin E1 and its main metabolites after intracavernous injection and short-term infusion of prostaglandin E1 in patients with erectile dysfunction. J Urol. 1997;158(4):1403-7.
14. Cox JW, Andreadis NA, Bone RC, Maunder RJ, Pullen RH, Ursprung JJ, et al. Pulmonary extraction and pharmacokinetics of prostaglandin E1 during continuous intravenous infusion in patients with adult respiratory distress syndrome. Am Rev Respir Dis. 1988;137(1):5-12. doi:10.1164/ajrccm/137.1.5.

15. Mizushima Y, Yanagawa A, Hoshi K. Prostaglandin E1 is more effective, when incorporated in lipid microspheres, for treatment of peripheral vascular diseases in man. J Pharm Pharmacol. 1983;35(10):666-7.

16. Gupta V, Rawat A, Ahsan F. Feasibility study of aerosolized prostaglandin E1 microspheres as a noninvasive therapy for pulmonary arterial hypertension. J Pharm Sci. 2010;99(4):1774-89. doi:10.1002/jps.21946.

17. Gupta V, Gupta N, Shaik IH, Mehvar R, Nozik-Grayck E, McMurtry IF, et al. Inhaled PLGA particles of prostaglandin E1 ameliorate symptoms and progression of pulmonary hypertension at a reduced dosing frequency. Mol Pharm. 2013;10(5):165567.

18. Gupta V, Davis M, Hope-Weeks LJ, Ahsan F. PLGA microparticles encapsulating prostaglandin E1-hydroxypropyl-betacyclodextrin (PGE1-HPbetaCD) complex for the treatment of pulmonary arterial hypertension (PAH). Pharm Res. 2011;28(7):1733-49. doi:10.1007/s11095-011-0409-6.

19. Ishihara T, Takahashi M, Higaki M, Takenaga M, Mizushima T, Mizushima Y. Prolonging the in vivo residence time of prostaglandin E1 with biodegradable nanoparticles. Pharm Res. 2008;25(7):1686-95.

20. Takenaga M, Ishihara T, Ohta Y, Tokura Y, Hamaguchi A, Igarashi R, et al. Nano PGE1 promoted the recovery from spinal cord injury-induced motor dysfunction through its accumulation and sustained release. J Control Release. 2010;148(2):249-54. doi:10.1016/j.jconrel.2010.08.003.

21. Takeda M, Maeda T, Ishihara T, Sakamoto H, Yuki K, Takasaki $\mathrm{N}$, et al. Synthesis of prostaglandin E1 phosphate derivatives and their encapsulation in biodegradable nanoparticles. Pharm Res. 2009;26(7):1792-800.

22. Gao Y, Xu P, Chen L, Li Y. Prostaglandin E1 encapsulated into lipid nanoparticles improves its anti-inflammatory effect with low side-effect. Int J Pharm. 2010;387(1):263-71.

23. Gao $\mathrm{F}$, Zhang $\mathrm{Z}, \mathrm{Bu} \mathrm{H}$, Huang $\mathrm{Y}$, Gao Z, Shen $\mathrm{J}$, et al. Nanoemulsion improves the oral absorption of candesartan cilexetil in rats: performance and mechanism. J Control Release. 2011;149(2):168-74. doi:10.1016/j.jconrel.2010.10.013.

24. Chen H, Khemtong C, Yang X, Chang X, Gao J. Nanonization strategies for poorly water-soluble drugs. Drug Discov Today. 2011;16(7-8):354-60. doi:10.1016/j.drudis.2010.02.009.

25. Ganta S, Singh A, Rawal Y, Cacaccio J, Patel NR, Kulkarni P, et al. Formulation development of a novel targeted theranostic nanoemulsion of docetaxel to overcome multidrug resistance in ovarian cancer. Drug Deliv. 2014:1-13. doi: 10.3109/ 10717544.2014.923068.

26. Sharma S, Sahni JK, Ali J, Baboota S. Effect of high-pressure homogenization on formulation of TPGS loaded nanoemulsion of rutin-pharmacodynamic and antioxidant studies. Drug Deliv. 2014. doi:10.3109/10717544.2014.893382.

27. Zhao H, Lu H, Gong T, Zhang Z. Nanoemulsion loaded with lycobetaine-oleic acid ionic complex: physicochemical characteristics, in vitro, in vivo evaluation, and antitumor activity. Int $\mathbf{J}$ Nanomedicine. 2013;8:1959-73. doi:10.2147/IJN.S43892ijn-8-1959.

28. Dordevic SM, Radulovic TS, Cekic ND, Randelovic DV, Savic MM, Krajisnik DR, et al. Experimental design in formulation of diazepam nanoemulsions: physicochemical and pharmacokinetic performances. J Pharm Sci. 2013;102(11):4159-72. doi:10.1002/ jps.23734.

29. Owens 3rd DE, Peppas NA. Opsonization, biodistribution, and pharmacokinetics of polymeric nanoparticles. Int J Pharm. 2006;307(1):93-102. doi:10.1016/j.ijpharm.2005.10.010.

30. Ichikawa H, Watanabe T, Tokumitsu H, Fukumori Y. Formulation considerations of gadolinium lipid nanoemulsion for intravenous delivery to tumors in neutron-capture therapy. Curr Drug Deliv. 2007;4(2):131-40.

31. Komori Y, Aiba T, Kushima M, Kawasaki H, Kurosaki Y. Alteration of therapeutic efficacy of lipid microspheres incorporating prostaglandin E1 by mixing with aqueous solution. J Pharm Sci. 2007;96(4):935-43. doi:10.1002/jps.20790. 
32. Ganta S, Talekar M, Singh A, Coleman TP, Amiji MM. Nanoemulsions in translational research-opportunities and challenges in targeted cancer therapy. AAPS PharmSciTech. 2014;15(3):694-708.

33. Kohli K, Chopra S, Dhar D, Arora S, Khar RK. Self-emulsifying drug delivery systems: an approach to enhance oral bioavailability. Drug Discov Today. 2010;15(21-22):958-65. doi:10.1016/ j.drudis.2010.08.007.

34. Choi HS, Liu W, Misra P, Tanaka E, Zimmer JP, Itty Ipe B, et al. Renal clearance of quantum dots. Nat Biotechnol. 2007:25(10):1165-70. doi:10.1038/nbt1340.

35. Contado C, Vighi E, Dalpiaz A, Leo E. Influence of secondary preparative parameters and aging effects on PLGA particle size distribution: a sedimentation field flow fractionation investigation. Anal Bioanal Chem. 2013;405(2-3):703-11. doi:10.1007/ s00216-012-6113-5.

36. Fang $\mathrm{C}$, Shi B, Pei YY, Hong MH, Wu J, Chen HZ. In vivo tumor targeting of tumor necrosis factor-alpha-loaded stealth nanoparticles: effect of MePEG molecular weight and particle size. Eur J Pharm Sci. 2006;27(1):27-36. doi:10.1016/ j.ejps.2005.08.002.

37. $\mathrm{Li}$ SD, Huang L. Pharmacokinetics and biodistribution of nanoparticles. Mol Pharm. 2008;5(4):496-504. doi:10.1021/ mp800049w.

38. Tang SY, Manickam S, Wei TK, Nashiru B. Formulation development and optimization of a novel Cremophore EL-based nanoemulsion using ultrasound cavitation. Ultrason Sonochem. 2012;19(2):330-45. doi:10.1016/j.ultsonch.2011.07.001.

39. Bian X, Liang S, John J, Hsiao CH, Wei X, Liang D, et al. Development of PLGA-based itraconazole injectable nanospheres for sustained release. Int J Nanomedicine. 2013;8:452131. doi:10.2147/IJN.S54040.

40. Sheng Y, Liu C, Yuan Y, Tao X, Yang F, Shan X, et al. Longcirculating polymeric nanoparticles bearing a combinatorial coating of PEG and water-soluble chitosan. Biomaterials. 2009;30(12):2340-8. doi:10.1016/j.biomaterials.2008.12.070.

41. Perry JL, Reuter KG, Kai MP, Herlihy KP, Jones SW, Luft JC, et al. PEGylated PRINT nanoparticles: the impact of PEG density on protein binding, macrophage association, biodistribution, and pharmacokinetics. Nano Lett. 2012;12(10):5304-10. doi:10.1021/nl302638g.
42. Ruiz A, Hernandez Y, Cabal C, Gonzalez E, VeintemillasVerdaguer S, Martinez E, et al. Biodistribution and pharmacokinetics of uniform magnetite nanoparticles chemically modified with polyethylene glycol. Nanoscale. 2013;5(23):11400-8. doi:10.1039/c3nr01412f.

43. Klibanov AL, Maruyama K, Torchilin VP, Huang L. Amphipathic polyethyleneglycols effectively prolong the circulation time of liposomes. FEBS Lett. 1990;268(1):235-7. doi:10.1016/00145793(90)81016-H

44. Moghimi SM, Hunter AC, Murray JC. Long-circulating and target-specific nanoparticles: theory to practice. Pharmacol Rev. 2001;53(2):283-318

45. Li SD, Huang L. Stealth nanoparticles: high density but sheddable PEG is a key for tumor targeting. J Control Release. 2010;145(3):178-81. doi:10.1016/j.jconrel.2010.03.016.

46. Schweer H, Meese CO, Watzer B, Seyberth HW. Determination of prostaglandin E1 and its main plasma metabolites 15-ketoprostaglandin E0 and prostaglandin E0 by gas chromatography/ negative ion chemical ionization triple-stage quadrupole mass spectrometry. Biol Mass Spectrom. 1994;23(3):165-70. doi:10.1002/bms.1200230308.

47. Hammes W, Buchsler U, Kinder P, Bokens H. Simultaneous determination of prostaglandin E1, prostaglandin E0 and 15-ketoprostaglandin E0 in human plasma by gas chromatography/ negative-ion chemical-ionization tandem mass spectrometry. J Chromatogr A. 1999;847(1-2):187-202.

48. Tsutsumiuchi R, Saito H, Imagawa T, Kitamori T, Odake T, Sawada T. In vivo determination of ultratrace amounts of prostaglandin in plasma by high-performance liquid chromatography/ laser-induced fluorometry/ultrasensitive laser spectrometry under severe conditions. Anal Chem. 1997;69(24):5006-10.

49. Zhang Y, Sun Y, Li G, Yin L, Wang T, Yang Y, et al. Determination of prostaglandin $\mathrm{E} 1$ in dog plasma using liquid chromatography-tandem mass spectrometry and its application to a pharmacokinetic study. J Chromatogr B. 2013;937:97-102. doi:10.1016/j.jchromb.2013.08.021.

50. Lin X, Zhang Y, Cui Y, Wang L, Wang J, Tang X. Determination of alprostadil in rat plasma by ultra performance liquid chromatography-electrospray ionization-tandem mass spectrometry after intravenous administration. J Pharm Biomed Anal. 2009;49(4):983-8. doi:10.1016/j.jpba.2009.01.019. 